\title{
ARTICULACIÓN DEL SISTEMA DE GESTIÓN INTEGRAL HSEQ BAJO LOS CRITERIOS DE NTC ISO 9001:2015, 14001:2015 Y 45001:2018 A LA EMPRESA COMPONENTES S.A.S.
}

\author{
${ }^{1}$ Paula Andrea Vargas Cuadrado Mdol, ${ }^{1}$ Edna Yohana Olivera Bermúdez. ${ }^{1}$ Erika \\ Dayana Chango Tupanteve \\ ${ }^{1}$ Universidad Autónoma De Colombia, Bogotá, Colombia
}

Recibido: 05/09/2021 Revisado: 03/10/2021 Aceptado: 16/11/2021 Publicado: 30/01/2022

\begin{abstract}
Resumen
El siguiente documento se evidencia la información actual de la organización en estudio Componentes S.A.S., su estado actual frente al Sistema de Gestión Integral desarrollado desde su creación y a través de los años. Este trabajo se ha realizado para verificar la conformidad de los criterios de evaluación de las Normas NTC ISO 9001:2015, 14001:2015 y 45001:2018, el nivel de cumplimiento de cada uno de estos criterios y la posible articulación de las mismas dentro de la organización y su mejoramiento continuo.
\end{abstract}

Se evidencia la importancia de la aplicación del sistema de gestión integral, ya que aporta en gran medida al funcionamiento objetivo de la empresa Componentes S.A.S., dando un valor agregado ante la competencia y aportando mejoras en los procesos internos y su competencia frente al mercado global.

Ahora bien, para realizar un análisis efectivo, es necesario tener la información real y verídica. Esto ayudará a evaluar la situación actual y recomendar los cambios pertinentes en búsqueda de la mejora continua. Al utilizar cada una de las herramientas para revisar y evaluar el nivel de cumplimiento para cada una de las Normas es de NTC ISO 9001:2015 (9\%), NTC ISO 14001:2015 (1\%) e NTC ISO 45001:2018 (3\%).

PALABRAS CLAVES: Sistemas, Gestión, Integral, HSEQ, ISO, Normas, auditoría, calidad, procedimientos, procesos, sistémico, modelo.

BIOTECH \& ENGINEERING Untels. Ene -Jun.2(1), 2022; ISSN:2788 -4295; 202-222 


\begin{abstract}
The following document shows the current information of the organization under study Components S.A.S., its current status against the Integral Management System developed since its creation and over the years. This work has been carried out to verify the conformity of the evaluation criteria of the NTC ISO 9001: 2015, 14001: 2015 and 45001: 2018 Standards, the level of compliance with each of these criteria and the possible articulation of the same within the organization and its continuous improvement.
\end{abstract}

The importance of the application of the integral management system is evident, since it contributes to a great extent to the objective operation of the company Componentes S.A.S. Giving added value to the competition and providing improvements in internal and external processes.

Now, to carry out an effective analysis, it is necessary to have real and truthful information. This will help to evaluate the current situation and recommend the pertinent changes in search of continuous improvement. When using each of the tools to review and evaluate the level of compliance for each of the Standards is NTC ISO 9001: 2015 (9\%), NTC ISO 14001:

$2015(1 \%)$ and NTC ISO 45001: $2018(3 \%)$. 


\section{INTRODUCCIÓN}

"Hoy mejor que ayer y mañana mejor que hoy"

Filosofía Kaizen

El proyecto de investigación; "Modelo de articulación del sistema de gestión integral HSEQ, bajo los criterios de las normas NTC ISO 9001:2015, NTC ISO 14001:2015 e NTC ISO 45001:2018”, pretende la integración de los Sistemas de Gestión de la Seguridad y Salud en el Trabajo, el Ambiental y de la Calidad - HSEQ Health, Safety, Environment, Quality). Esta integración se ha c(onvertido en una tendencia en el presente siglo, incrementándose en los años recientes, como una necesidad de implementarlos en las empresas.

Desde el punto de vista de la normalización que ha establecido la NTC ISO (Organización internacional de normalización). Se considerarán las normas: NTC ISO 9001:2015. Sistemas de gestión de la calidad. Requisitos; NTC ISO 14001: 2015. Sistema de gestión ambiental. Requisitos con orientación de uso e NTC ISO 45001:2018. Sistemas de gestión de seguridad y salud en el trabajo. Requisitos con orientación para el uso. Estas normas, obedecen a la estructura de alto nivel establecida en el anexo SL contenido en el suplemento NTC ISO consolidado que contiene los procedimientos oficiales que establecen cómo desarrollar una norma NTC ISO. El propósito es articular estas normas con la legislación colombiana, así como con los modelos de excelencia de calidad más relevantes.

Para alcanzar un mayor resultado en la integración de los sistemas de gestión, se pretende incluir otro punto de vista. Desde la cibernética organizacional y en especial desde el modelo de sistema viable de Stafford Beer, establecer la viabilidad del sistema, así como identificar los obstáculos de aprendizaje utilizando el modelo OADI-SMM e identificar arquetipos organizaciones. El propósito es asegurar la viabilidad del sistema integrado superando los obstáculos de aprendizaje y los arquetipos organizacionales.

Para lograr cubrir los propósitos se constituyó el Semillero de Investigación HSEQ compuesto por estudiantes de programas de pregrado de la Universidad, quienes están optando a su grado profesional.

BIOTECH \& ENGINEERING Untels. Ene -Jun.2(1), 2022; ISSN:2788 -4295; 202-222 
En este informe de investigación realizado por estudiantes del programa de Ingeniería Industrial, tiene como objetivo proponer una articulación del Sistema de Gestión Integral HSEQ como aporte al propósito del proyecto de investigación.

El aseguramiento de la satisfacción del cliente, la mejora continua y el concepto de calidad ha tomado mayor fuerza a nivel regional, ya que se ha convertido en una necesidad, que más allá de ser un valor agregado, se ha vuelto una estrategia de competencia y reconocimiento con fines comerciales. Así las cosas, las empresas han identificado la importancia de implementar su Sistema de Gestión Integral, con el fin de garantizar que todos sus procesos cumplan con estándares mínimos, no solo en términos de calidad, sino de la administración de la salud, seguridad y medio ambiente.

Un Modelo de Sistema Viable es utilizado como medio para diagnosticar el funcionamiento de las Organizaciones y su diseño en busca de la mejora de las capacidades organizacionales, la interpretación de sus diferentes funciones con el fin de generar estabilidad y adaptabilidad que, sin lugar a dudas, trae el crecimiento efectivo dentro de una organización.

Según Stafford Beer, una empresa es un "sistema dinámico y superviviente". Cuando se refiere a que es un sistema se entiende que es una interrelación entre diferentes partes y dinámico porque evolucionan continuamente. Además, indica que existen defectos y efectos adversos dentro de la organización que ocasionan inestabilidad y que una de sus razones es la adaptabilidad a los diversos factores existentes.

Por otro lado, el Modelo Europeo de Excelencia Empresarial desarrollado por EFQM (Fundación Europea para la Gestión de la Calidad), es una herramienta orientada en la búsqueda de la mejora continua de su gestión hacia la excelencia, integrando estándares de aseguramiento de la calidad y eficiencia en procesos conocidas como las NTC ISO.

El Modelo de Malcom Baldrige, también es reconocido por su importancia para evaluar el Sistema de Gestión de Calidad de una organización, teniendo en cuenta aspectos importantes tales como el Liderazgo, Planificación Estratégica, Gestión de Procesos, entre otros, orientada hacia la obtención de resultados y creación de valor.

BIOTECH \& ENGINEERING Untels. Ene -Jun.2(1), 2022; ISSN:2788 -4295; 202-222 
Actualmente, en Colombia existe un reconocimiento a las buenas prácticas de gestión de calidad conocido como premio colombiano a la Calidad, en donde busca articular los procesos con el fin de mantenerse vigente en un mercado global.

Para la empresa privada se ha convertido una necesidad contemplar la implementación de un Sistema de Gestión Integral para el fortalecimiento de la organización, desde un contexto interno y externo, y para lograr un valor agregado y diferenciador frente a su competencia. En Colombia, según el Ministerio de Comercio, Industria y Turismo y Colombia Productiva, 6 de cada 10 empresas no cuentan con ningún tipo de certificación de calidad.

\section{OBJETIVOS}

\subsection{OBJETIVO GENERAL}

Proponer una articulación del sistema de gestión integral HSEQ bajo los criterios de las normas NTC ISO 9001:2015, NTC ISO 14001: 2015 y NTC ISO 45001:2018.

\subsection{OBJETIVOS ESPECÍFICOS}

- Aplicar los instrumentos diagnósticos para establecer el estado actual de la empresa en el sistema de gestión integral HSEQ. (Desde las normas NTC ISO y desde el MSV).

- Establecer el plan de mejora para la implementación del sistema integral de gestión HSEQ.

- Determinar el alcance del uso de los instrumentos diagnósticos para formular el plan de mejora. ¿Qué tanto contribuyeron los instrumentos para poder formular un plan de mejora? ¿Qué dificultades se presentaron al momento de aplicar los instrumentos diagnósticos?

BIOTECH \& ENGINEERING Untels. Ene -Jun.2(1), 2022; ISSN:2788 -4295; 202-222 


\section{PRESENTACIÓN DE LA EMPRESA}

Componentes S.A.S., es una organización legalmente constituida desde marzo de 2018, como iniciativa de una pareja de esposos que quisieron crear su propia empresa de acuerdo a la experiencia generada al trabajar en diferentes sectores de la economía y después de tener una sociedad por alrededor de 4 años en una empresa similar que fue liquidada hace un año. Sus instalaciones físicas están en la localidad de Suba en la Carrera 100B \#153-44, Bogotá D.C

- Cundinamarca, Suba - Pinar. Iniciaron con una idea de negocio, pero a través de los años han ampliado su portafolio de servicios con nuevas líneas de negocio para poder satisfacer las necesidades del mercado actual.

Esta pareja de socios - esposos, a través de la experiencia que han adquirido durante el tiempo, decidieron crear COMPONENTES S.A.S., teniendo en cuenta que sus perfiles profesionales se convertían en una gran herramienta, pues uno de ellos es egresado de la Fundación Universidad Autónoma de Colombia FUAC de la carrera de Ingeniería Industrial y su esposa es Administradora de Empresas.

Dentro de su alcance, la empresa se dedica a comercializa Autopartes, línea de ferretería, herramientas manuales y consumibles, Elementos de protección personal EPP's y Bioseguridad, además de realizar mantenimiento y recarga de equipos de extinción (extintores).

El crecimiento de la organización se ha generado por la ampliación de su portafolio de sus productos y servicios, pues se han visto en la necesidad de ingresar en nuevos mercados con el fin de obtener reconocimiento y poder satisfacer las necesidades de sus clientes.

A través de los años se han visto en la necesidad de implementar su Sistema de Gestión Integral por las múltiples exigencias que les ha traído la inmersión a un mercado más fuerte como el Sistema de Transporte Masivo Transmilenio y otras entidades que dentro de sus requisitos internos, han solicitado a sus proveedores contar con su sistema implementado. Sin embargo, la empresa ha trabajado de manera empírica durante sus años de trabajo y aunque han considerado el levantamiento de su sistema, más que una oportunidad de crecimiento y fuerte herramienta de sostenimiento, se han visto obligados a ser parte lo antes posible del mundo empresarial moderno relacionados con Sistemas de Gestión Integral. 
Su metodología organizacional se ha basado en lo empírico y ha tomado gran importancia, puesto que así se ha mantenido desde la apertura de la organización; su área estratégica, conformada por el Gerente Comercial y la Gerente Administrativa y Comercial, han desarrollado habilidades que les ha traído el diario vivir; conocen en absoluto sus clientes potenciales así como sus proveedores más frecuentes; sin embargo, todo este conocimiento y manejo de la empresa no ha sido documentado, situación que incumple uno de los criterios fundamentales o debes de las normas que es el documentar todos los procesos; todo esto, con el fin de contar con una evidencia objetiva del desarrollo de sus actividades, y en ese sentido, conocer y comprender mejor la misma.

Dentro de sus intentos por implementar su sistema de gestión, se ha logrado identificar algunos de sus procesos y manuales, así como la identificación de una estructura organizacional, que durante la etapa de verificación se evidenció el desconocimiento de los documentos existentes a la fecha; situación que no garantiza la mejora continua, puesto que el intento por levantar su sistema de gestión se ha visto opacado por su falta de constancia.

La organización ha podido demostrar compromiso en las diferentes actividades que salen del día a día, evidenciando participación activa y alta pro-actividad por parte del área de estrategia a cargado de los dueños; sin embargo, este trabajo en conjunto no ha permitido que la empresa busque la manera adecuada de asignar roles y responsabilidades con el fin de crear su sistema de gestión, pues todas la tareas y actividades quedan en cabeza de la alta gerencia. Actualmente, no cuentan con el suficiente personal para asignar responsabilidades y cuando se incorpora a alguien para llevar una tarea no existe continuidad y las mismas se quedan en espera y muchas de ellas sin terminar.

A causa de lo anterior, la organización durante los 3 años que lleva de ejecución ha tenido varios intentos por implementar su sistema de gestión, pero no han tenido el éxito esperado, pues no han podido definir concretamente los responsables por el alto flujo y deserción de personal.

\section{MARCO TEÓRICO}

\subsection{Ciclo PHVA (Planificar- Hacer- Verificar- Actuar)}


Las listas de verificación están alineadas con el ciclo Deming PHVA (Planificar- HacerVerificar- Actuar), el cual desarrolla una metodología lógica que aporta a un buen desarrollo de los sistemas de gestión aplicado a cualquier ámbito ya sea el de calidad, ambiental o en seguridad y salud en el trabajo.

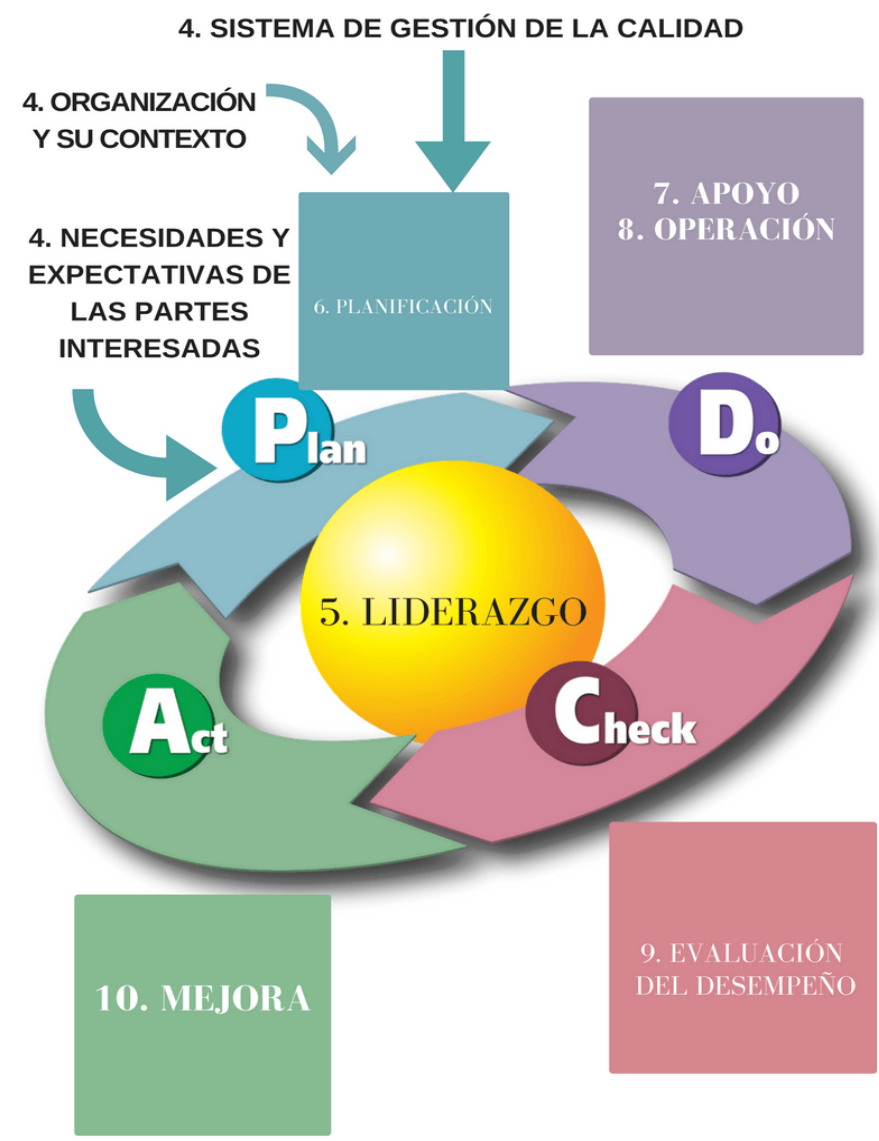

Figura 1 Ciclo Demming

Nota. Recuperado:Pág web https://aprendiendocalidadyadr.com/ciclo-deming-en-la-NTCISO-90012015/

Estas cuatro palabras han significado mucho para el mejoramiento continuo de las organizaciones, ya que sintetiza o guía en la ejecución de una acción o la ejecución controlada de lo planificado, con el fin de, una vez se efectúe lo ejecutado, revisarlo y ajustarlo si es 
necesario. El sentido fundamental del ciclo, es que el mismo vuelve a iniciar una planificación y éste nunca terminará; de ahí que el Ciclo de Deming PHVA, conocido también por sus siglas en inglés como PDCA (Plan- Do- Check- Act), conlleve a las organizaciones hacia la mejora continua. (Gehisy, 2016)

\subsection{Listas de verificación NTC ISO 9001:2015, NTC 14001:2015 y NTC ISO 45001:2018}

Las listas de verificación de las normas están orientadas para el análisis inicial de las organizaciones, es decir, la verificación de su estado actual. Estas listas se construyeron a partir de la investigación y articulación de diferentes metodologías.

La articulación de la Lista de Verificación NTC ISO 9001:2015 (Contreras Vallecilla, J, A., Moreno Huertas, W, A., \& Soriano Novoa, D, M., 2020), se encuentra bajo el concepto de estructura de alto nivel, la cual cuenta con introducción, alcance, referencias normativas, términos y definiciones, contexto de la empresa, liderazgo, planificación, soporte, operaciones, evaluación del desempeño y mejora.

Estos aspectos generan un lineamiento coherente en búsqueda de la eficiencia y eficacia, el desarrollo interno y externo de las organizaciones y de igual manera coexisten otras metodologías como el Modelo Europeo de Excelencia Empresarial desarrollado por EFQM (Fundación Europea para la Gestión de la Calidad); en este modelo se establecen dos agentes que permiten evaluar si la organización cumple con los estándares de excelencia definidos.

Inicialmente se relacionan 9 criterios clasificados como agentes facilitadores los cuales son: liderazgo, estrategia, recursos humanos, procesos, alianzas externas y recursos internos; seguidamente los agentes de resultado que se clasifican en: empleados, clientes, la sociedad y resultados clave, logrando con esto innovación y mejora en las organizaciones.

Malcolm Baldrige diseño el modelo de excelencia que se basa en la realización de autoevaluaciones dentro de la estructura organizativa que proporciona retroalimentación a la misma, en este modelo se desarrollan tres enfoques que fortalecen la competitividad los cuales son: ayudar a mejorar sus prácticas de gestión, capacidades y resultados, facilitar la comunicación y el intercambio de información sobre mejores prácticas entre organizaciones de todo tipo y servir de herramienta de trabajo para comprender y gestionar el desempeño la planificación y las oportunidades de aprendizaje. 
El premio colombiano es una de las últimas metodologías y no menos importante, utilizada en la articulación de las listas de verificación, este modelo se enfoca en la competitividad, innovación y aprendizaje. Se deben desarrollar para lograr un éxito sostenible; en el desarrollo es importante resaltar las buenas prácticas empresariales, orientadas a un constante cambio con respecto a las acciones de mejora que se establezcan durante el paso del tiempo buscando la excelencia organizacional en cada proceso que se tiene establecido.

Estas metodologías están orientadas en búsqueda de la calidad y excelencia total dentro de la organización, la composición de estas metodologías en la lista de verificación, dará un mayor alcance a todos los niveles organizacionales, dando un valor agregado en el momento de aplicar este modelo a una compañía, logrando tener un análisis completo del estado actual de la organización y generar planes de acción para la mejora continua.

Por otro lado, la lista de verificación NTC ISO 14001:2015 Camberos Ortiz, M, E. \& Higuera Barbosa, G, S. (21 de 11 de 2020) contiene la estructura de alto nivel definida para la familia de las normas NTC ISO, está enfocada en la validación de los aspectos ambientales que se desarrollan en las organizaciones en búsqueda de minimizar los impactos ambientales que se pueden generar en el desarrollo de los procesos misionales.

El objetivo de esta lista de verificación es analizar cada uno de los parámetros establecidos en la norma en mira de demostrar la responsabilidad en la protección del medio ambiente, implementación o mejora del sistema de gestión ambiental.

Los beneficios que se obtienen al desarrollar esta lista de verificación, es conocer dentro de la organización que proceso está impactando ambientalmente, y como valor agregado brindar beneficios económicos y fortalecer la imagen de la organización en cuanto a sostenibilidad ambiental.

La metodología que se utilizó en la lista de verificación NTC ISO 45001:2018 (Bareño Moreno, H, K., López Rodríguez, Y, M., \& Prieto Ramírez, L, F., 2020), es en la búsqueda de la protección de los colaboradores en una organización minimizando los riesgos y/o peligros que se pueden generar por los procesos en el desarrollo de sus funciones, evitando accidentes, incidentes y enfermedades laborales.

\subsection{Arquetipos Organizacionales:}

BIOTECH \& ENGINEERING Untels. Ene -Jun.2(1), 2022; ISSN:2788 -4295; 202-222 
La cultura organizacional se ha convertido en un tema referente para las organizaciones, por ende, la integración de los arquetipos organizacionales con las normas NTC ISO 9001:2015, NTC ISO 14001:2015 y NTC ISO 45001:2018, busca la identificación de las actitudes, comportamientos y conductas que tiene un individuo, grupo o sociedad y que pueden impactar a una organización caracterizada por el pensamiento sistémico.

Para dicho análisis se aplicó la Herramienta para Identificar Arquetipos Organizacionales (Maldonado Méndez, J, S., Suárez Russi, Y, M., \& Vargas Medina, L, N.). Identificando la relación existente entre los cuatro elementos de la quinta disciplina: dominio personal, modelos mentales, visión compartida y aprendizaje en equipo por (Senge, 2010) y los cuatro aspectos para formar una organización recursiva: identidad, cohesión, ciudadanía y desempeño para comprender el sistema recursivo de una organización por (Espejo, 1999).

\subsection{Obstáculos de aprendizaje:}

En algunas ocasiones el desempeño de la organización se ve afectado por los obstáculos de aprendizaje que se pueden generar con el pasar del tiempo; reduciendo la capacidad competitiva que tiene una organización frente a las demás, cabe aclarar que estos obstáculos se pueden identificar de manera individual $u$ organizacional afectando igualmente a los colaboradores.

Por lo tanto, se hace necesario la identificación de los obstáculos que puedan afectar a la organización y por ende poder atacarlos asertivamente. Hay siete tipos de obstáculos identificados por (Espejo, 1999) para la identificación se aplicó la Herramienta Diagnóstica

- Obstáculos de Aprendizaje (Guevara Cerón, G, R., Jiménez Mora, M, L., \& Rodríguez Moreno, J, T., 2020).

\subsection{Modelo de Sistema Viable MSV}

El Modelo de Sistema Viable MSV, es utilizado como medio para diagnosticar el funcionamiento de las Organizaciones y su diseño. Las mejoras de las capacidades organizacionales, la interpretación de sus diferentes funciones con el fin de generar estabilidad y adaptabilidad que trae consigo el crecimiento efectivo. 
Según el Stafford Beer, "La estructura organizacional de cualquier empresa no es solo relaciones de autoridad y control; es, además, el conjunto de normas, acuerdos y cultura inherente a la organización misma, y que inciden en el comportamiento esperado de la organización. Son estos últimos, quienes bloquean o inhiben el cambio" (Rueda, 1995). Cuando se refiere a que es un sistema se entiende que es una interrelación entre diferentes partes y que además es dinámico porque evolucionan continuamente. Además, indica que existen defectos dentro de la organización que ocasionan inestabilidad y que unas de sus razones es la adaptabilidad a los diversos factores existentes.

También explica el porqué del fracaso de algunas organizaciones, entre ellas se considera la interpretación errada de las herramientas disponibles que permiten la solución de problemas y ayudan a sobrevivir dentro de un entorno.

Existen tres factores o entidad básica con las que cuenta un Sistema Viable; Entorno, Operación y Gestión. El primero hace referencia al sector económico en donde opera o se encuentra una organización; además de factores tales como sociales y políticos que afectan o condicionan dicha organización. Cuando se refiere a Operación, es todo aquello que produce internamente el Sistema y le da un alcance a su actividad. Y, por último, está la Gestión, que son las actividades de dirección o cabeza principal necesaria para hacer funcional el sistema. A diferencia de la Operación, la Gestión no puede existir por sí misma.

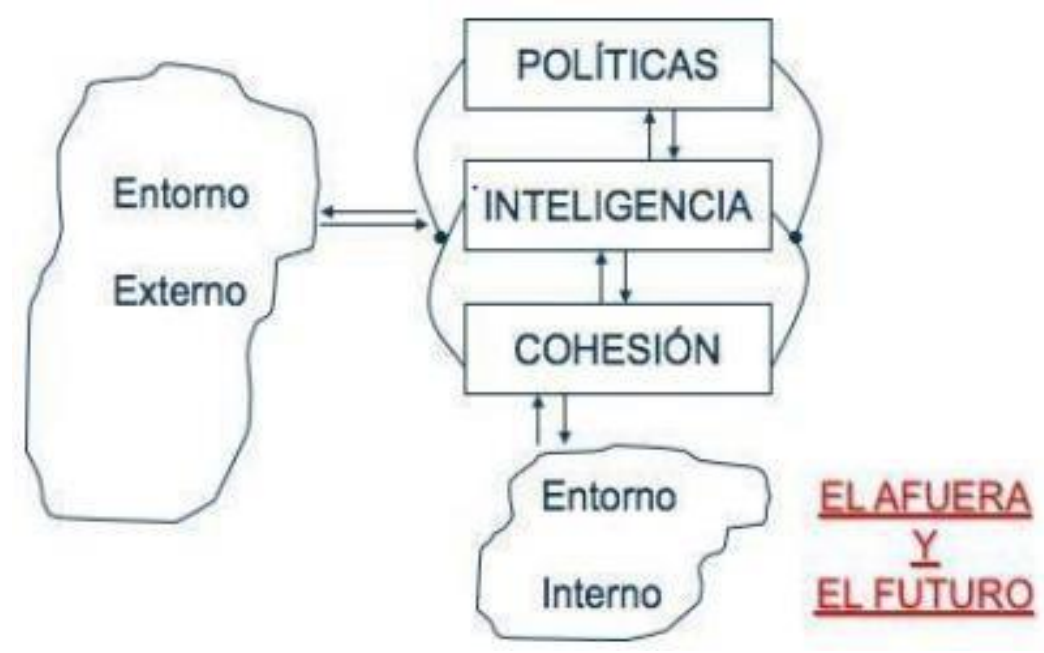

Figura 2 Mecanismo de adaptación

Nota. Tomado del semillero de Investigación HSEQ, por Pérez, G. 2020. Adaptado de Espejo, R. 2003 The Viable System Model. A briefing about organizational struture. Syncho Limited. 


\section{DESARROLLO}

\subsection{Metodología para la aplicación de las Listas de Verificación}

La aplicación de las listas se efectuó de manera remota debido a la coyuntura ocasionada por la Pandemia de la COVID -19. Se diseñó un plan de trabajo con una metodología organizada y secuencial para la aplicación de cada uno de los capítulos de las listas de verificación teniendo como base la Norma NTC ISO 19011:2018 Directrices para la auditoría de los sistemas de gestión.

El plan de trabajo se compone de una información básica como objetivo de la auditoría, alcance, roles y responsabilidades. Adicionalmente, se relaciona un campo llamado actividades, en donde se detalló el tiempo que se establecía para la ejecución de dicha actividad y los recursos necesarios para llevarla a cabo el evento.

Cada encuentro tuvo una duración de 1 hora y 30 minutos, dando inicio a las 5:15 pm y finalizando a las 6:45 pm, los días viernes de cada semana.

La metodología de la auditoría se llevó a cabo por la solicitud de documentos o registros que sustenten el apartado relacionado en el plan de trabajo que se compartía vía correo electrónico cada semana a la empresa Componentes S.A.S.

Como dinámica, en cada plan de trabajo era asignado un líder que estaba encargado de organizarlo y enviarlo para la semana correspondiente, coordinar y dirigir el encuentro, orientando al responsable auditado en cada capítulo, indicando lo que solicita la norma y cómo podría cumplir ese requerimiento.

Por otro lado, la empresa Componentes S.A.S., compartió el dominio de la plataforma Drive, a través de su correo electrónico, en donde se encuentran cargados los documentos soporte que sustentan el trabajo que ha realizado la empresa referente al sistema de gestión integral. La empresa en cumplimiento a la Ley 1581 de 2012 de protección de datos realizó una cláusula de confidencialidad con cada uno de los participantes de la aplicación de las listas en verificación. 
Se organizó un encuentro remoto con el gerente y dueño de la empresa Componentes S.A.S., donde se dio a conocer el alcance de la propuesta de articulación HSEQ, explicando la metodología que se llevaría a cabo y la manera de recolectar la información. Por medio de una presentación en diapositivas se informó acerca del modelo del sistema viable y los aportes a la implementación del SGI.

Adicionalmente, se resaltaron los beneficios que tendría la empresa al realizar la articulación del sistema en la organización.

Al realizar la recolección de la información se evidencio el estado actual de la empresa Componentes S.A.S.

\subsection{Interpretación general Listas de Verificación}

La interpretación de los resultados, para la lista de verificación NTC ISO 9001:2015 (Contreras Vallecilla, J, A., Moreno Huertas, W, A., \& Soriano Novoa, D, M., 2020), tiene como resultado definitivo para la organización "sólidamente fundamentado-bajo"; encontrando varios procesos definidos y documentados que presentan falencias en su estructura, actualización o simplemente no existen. Mostrando debilidad en las acciones de mejora requeridas por la norma.

La interpretación de los resultados, arrojó una calificación en el ítem de planificación del ciclo PHVA (Planificar -Hacer-Verificar-Actuar), que para este caso serían los capítulos 4,

5 y 6 de las normas objeto de estudio. sólidamente fundamentado bajo evidenciando un

conocimiento básico de los procesos y del contexto general a nivel calidad y ambiental de la organización, los cuales tiene un nivel mínimo documentado. Cabe resaltar que la empresa se encuentra legalmente constituida, identifica la competencia, la estructura organizacional está definida sin embargo no es claro cuáles son los procesos estratégicos, misionales y de apoyo aplicables lo cual no concuerda con el alcance establecido por la organización. No existe una metodología documentada adecuada para la revisión y actualización de documentos.

Dentro del sistema de seguridad y salud en el trabajo SG-SST tiene predeterminados y creados algunos documentos de requisitos legales mínimos establecidos por el Ministerio 
de Trabajo tales como el Decreto 1072 de 2015, Decreto Único Reglamentario del Sector Trabajo, por lo que se ha tenido que regir bajo estos decretos por los múltiples requerimientos del mercado, pero sin tener en cuenta el cumplimiento de los requisitos de la norma NTC ISO 45001:2018, que si bien en algunos aspectos se alinean no cumplen en su totalidad con los criterios directos de la norma.

En el siguiente ítem referente al Hacer del ciclo PHVA (Planificar -Hacer-Verificar-Actuar), el cual corresponde a los capítulos 7 y 8 de las normas objeto de estudio. Se evidencia para el capítulo 7 que algunos procesos misionales y de apoyo de la organización se encuentran identificados y establecidos, aunque para el tema de los recursos la empresa informa que ha asignado los recursos necesarios y el personal idóneo para el desarrollo de las actividades, pero no se cuenta con ningún soporte o evidencia que permita conocer el análisis realizado y la metodología utilizada para obtener dicha información.

Por otro lado, desde que se implementaron las diferentes listas de verificación, se evidencia que la organización no realiza ningún tipo de diseño, creación, fabricación o transformación de materia prima, exonerándolos del requisito 8.3 del capítulo 8 de la norma. Bajo el numeral 8 de la norma NTC ISO 14001:2015 y de NTC ISO 45001:2018 (Bareño Moreno, H, K.,

López Rodríguez, Y, M., \& Prieto Ramírez, L, F., 2020), no se encontró evidencia alguna ya que el nivel de cumplimiento de estos criterios es nulo.

Para el ítem de Verificación del ciclo PHVA (Planificar -Hacer-Verificar-Actuar), para el capítulo 9 de las normas objeto de estudio, al no contar con una definición concreta de los capítulos anteriores, la organización no tiene establecido que es lo que debe evaluar para llegar a la mejora continua, incumplimiento los criterios establecidos como son la auditoría interna y el seguimiento necesario que debe realizar la alta dirección.

Finalmente, dentro del ítem de Actuar del ciclo PHVA (Planificar -Hacer-Verificar-Actuar), correspondiente al capítulo 10 de las normas objeto de estudio, al no definir los anteriores capítulos de las normas, la mejora continua se vuelve difícil de identificar dentro de la organización.

\subsection{Interpretación de resultados aplicación listas de verificación NTC ISO 9001:2015}

Con la aplicación de la Lista de Verificación NTC ISO 9001:2015 (Contreras Vallecilla, J, A., Moreno Huertas, W, A., \& Soriano Novoa, D, M., 2020) se comprobó, que, aunque tiene 
un mayor cumplimiento frente a las demás normas, la organización cuenta con un mínimo de implementación de su Sistema de Gestión de Calidad.

Como hemos visto en líneas anteriores, la organización no tiene identificado apropiadamente su contexto organizacional, lo que en principio conlleva a un fuerte impacto en la adecuada gestión en las diferentes actividades propias de la organización.

La información interna y externa considerada útil e importante para la organización es manejada por la alta dirección, pero no hay documentación ni divulgación dentro de la organización, lo que, no les ha permitido establecer objetivos y acciones en pro de la mejora y el buen desempeño de la misma.

El área estratégica, conformada por la Gerente Administrativa y Financiera y su socio el Gerente Comercial, cuentan con un alto desarrollo en aspectos relacionados con liderazgo organizacional, lo que les ha permitido crear una buena imagen corporativa dentro de su nicho de mercado; sin embargo, los diferentes procesos y actividades de la organización han quedado en cabeza de ellos mismos, lo que no les ha permitido desarrollar estrategias competitivas para abordar los cambios del mercado en general.

Existe poca información documentada. En sus intentos por implementar su Sistema de Gestión de Calidad han desarrollado planes de trabajo que al final no se ejecutan porque no contienen el sustento apropiado. Las áreas de operación no cuentan con el personal suficiente para garantizar el apropiado funcionamiento. Por lo anterior el resultado final es $8 \%$ - SÓLIDAMENTE FUNDAMENTADO - BAJO.

\begin{tabular}{|l|r|l|}
\hline \multicolumn{3}{|c|}{ CUMPLIMIENTO POR CAPITULOS } \\
\hline 4. CONTEXTO DE LA ORGANIZACIÓN & $7 \%$ & SÓLIDAMENTE FUNDAMENTADO - BAJO \\
\hline 5. LIDERAZGO Y PARTICIPACIÓN DE LOS & $13 \%$ & SÓLIDAMENTE FUNDAMENTADO - MEDIO \\
\hline TRABAJADORES & & \\
\hline 6. PLANIFICACIÓN & $15 \%$ & SÓLIDAMENTE FUNDAMENTADO - MEDIO \\
\hline 7. APOYO & $10 \%$ & SÓLIDAMENTE FUNDAMENTADO - MEDIO \\
\hline 8. OPERACIÓN & $7 \%$ & SÓLIDAMENTE FUNDAMENTADO - BAJO \\
\hline 9. EVALUACIÓN DE DESEMPEÑO & $1 \%$ & SÓLIDAMENTE FUNDAMENTADO - BAJO \\
\hline 10.MEJORA & $0 \%$ & NO EXISTE \\
\hline
\end{tabular}

Tabla No. 1 
Tabla de resultados nivel de cumplimiento ISO 9001:2015 por capítulos

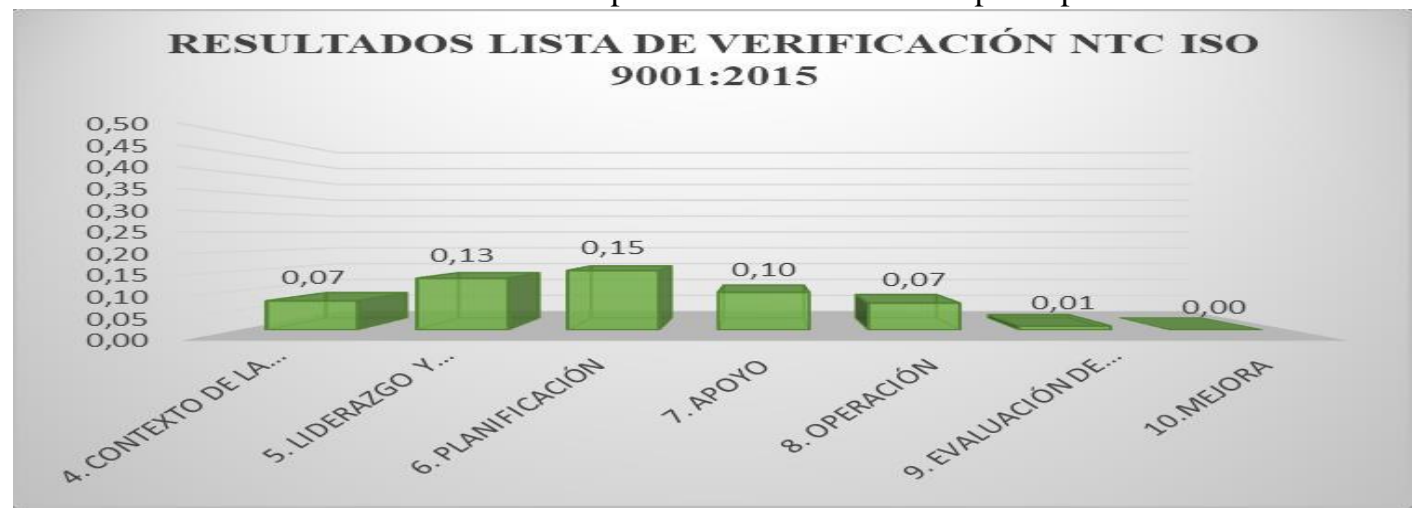

Gráfica No.1

Gráfica resultados nivel de cumplimiento ISO 9001:2015 por capítulos

\subsection{Interpretación de resultados aplicación listas de verificación NTC ISO 14001:2015}

Bajo la aplicación de la Lista de Verificación NTC ISO 14001:2015, se evidenció que el nivel de cumplimiento de los requisitos de la norma es casi nulo, puesto que, en relación con aspectos e impactos medioambientales, la organización no ha tomado gestión alguna para abordarlos. No existe evidencia de información documentada, ni análisis frente a las responsabilidades con el medio ambiente generas a partir de su operación.

La organización ha manifestado en diferentes ocasiones que para la prestación de su servicio se ha convertido en una gran necesidad implementar su Sistema de Gestión Ambiental para cumplir y reforzar su imagen comercial frente a sus clientes potenciales y así efectuar una venta o prestar un servicio en el futuro, lo que se le ha convertido en una necesidad y un proyecto a corto plazo.

Por lo anterior el resultado final es $1 \%$ - SÓLIDAMENTE FUNDAMENTADO - BAJO.

\begin{tabular}{|l|c|l|}
\hline \multicolumn{3}{|c|}{ CUMPLIMIENTO POR CAPÍTULOS } \\
\hline 4. CONTEXTO DE LA ORGANIZACIÓN & $3 \%$ & SÓLIDAMENTE FUNDAMENTADO - BANO \\
\hline 5. LIDERAZGO Y PARTICIPACIÓN DE LOS TRABAJADORES & $0 \%$ & NO EXISTE \\
\hline 6. PLANIFICACIÓN & $0 \%$ & NO EXISTE \\
\hline 7. APOYO & $2 \%$ & SÓLLDAMENTE FUNDAMENTADO - BANO \\
\hline 8. OPERACIÓN & $0 \%$ & NO EXISTE \\
\hline 9. EVALUACIÓN DE DESEMPENOO & $0 \%$ & NO EXISTE \\
\hline 10.MEORA & $0 \%$ & NO EXISTE \\
\hline
\end{tabular}


Tabla No. 2

Tabla de resultados nivel de cumplimiento ISO 14001:2015 por capítulos

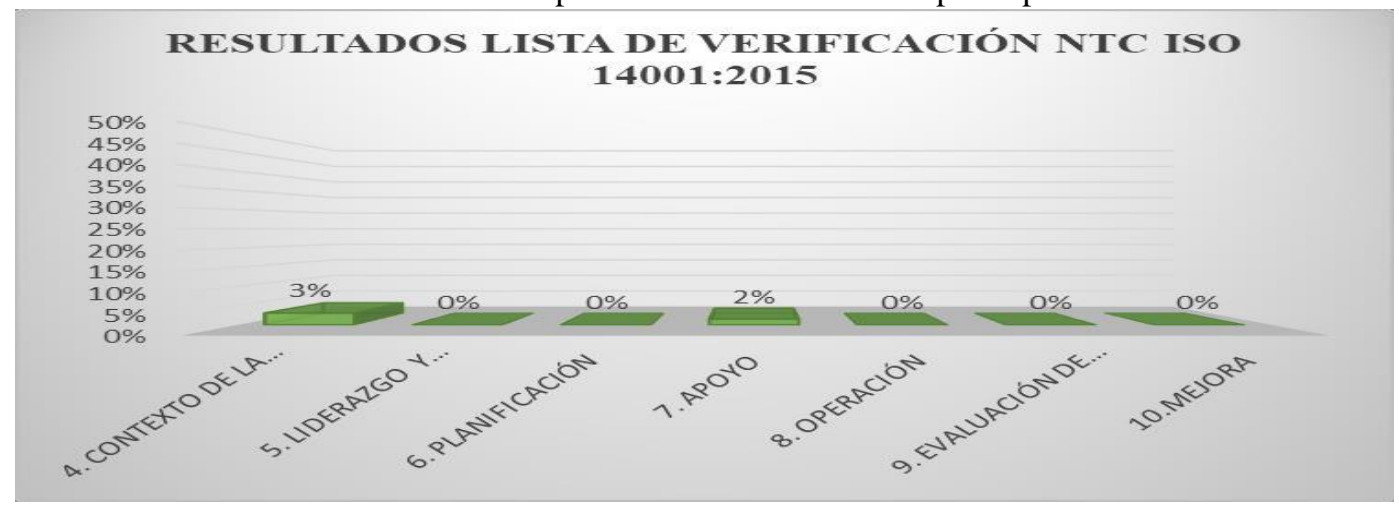

Gráfica No.2

Gráfica resultados nivel de cumplimiento ISO 14001:2015 por capítulos

\subsection{Interpretación de resultados aplicación listas de verificación NTC ISO 45001:2018}

Tras hacer aplicación de la lista de verificación NTC ISO 45001:2018 (Bareño Moreno, H, K., López Rodríguez, Y, M., \& Prieto Ramírez, L, F., 2020) para la empresa Componentes S.A.S., se identificó que el cumplimento del Sistema de Seguridad y Salud en el trabajo SGSST no está definido, esto evidencia que la organización no cuenta con una planeación que conlleve a la ejecución del mismo, el cual beneficia tanto a la empresa como a sus colaboradores al evitar accidentes de trabajo o enfermedades laborales.

La organización cuenta con cierta documentación, pero no está alineada normativamente generando cumplimiento frente al decreto 1072 de 2015 es cual es obligatorio ante el Ministerio de Trabajo, por una parte y por otra el nivel de cumplimiento ante los requisitos de la Norma ISO 45001:2015 es baja.

En cuanto al resultado de la lista de verificación es SÓLIDAMENTE FUNDAMENTADO BAJO con un porcentaje de cumplimento del $3 \%$. 


\begin{tabular}{|l|r|l|}
\hline \multicolumn{3}{|c|}{ CUMPLIMIENTO POR CAPÍTULOS } \\
\hline 4. CONTEXTO DE LA ORGANIZACIÓN & $11 \%$ & SÓLIDAMENTE FUNDAMENTADO - MEDIO \\
\hline 5. LIDERAZGO Y PARTICIPACIÓN DE LOS TRABAJADORES & $5 \%$ & SÓLIDAMENTE FUNDAMENTADO - BAJO \\
\hline 6. PLANIFICACIÓN & $5 \%$ & SÓLIDAMENTE FUNDAMENTADO - BAJO \\
\hline 7. APOYO & $1 \%$ & SÓLIDAMENTE FUNDAMENTADO - BAJO \\
\hline 8. OPERACIÓN & $0 \%$ & NO EXISTE \\
\hline 9. EVALUACIÓN DE DESEMPEÑO & $0 \%$ & NO EXISTE \\
\hline 10.MEJORA & $0 \%$ & NO EXISTE \\
\hline
\end{tabular}

Tabla No. 3

Tabla de resultados nivel de cumplimiento ISO 45001:2018 por capítulos

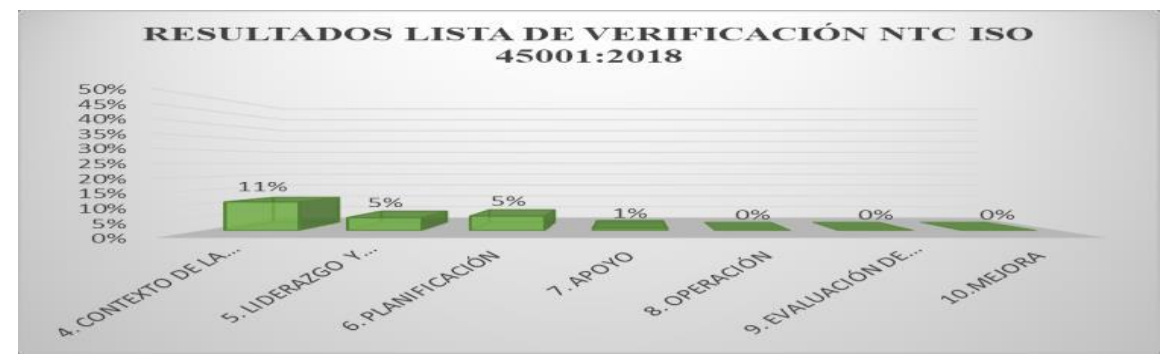

Gráfica No. 3

Gráfica resultados nivel de cumplimiento ISO 45001:2018 por capítulo

\subsection{Interpretación de herramienta de Arquetipos Organizacionales}

La quinta disciplina de (Senge, 2010) hace referencia a un pensamiento sistémico, incluyendo los cuatro aspectos de (Espejo, 1999)sobre un sistema recursivo; permiten evaluar en la empresa Componentes S.A.S., la gestión de arquetipos de la siguiente manera:

Identidad // Dominio personal: La empresa Componentes S.A.S., tiene definidos algunos de sus procesos misionales y operativos; sin embargo, no es suficiente para garantizar un control adecuado que permita analizar y tomar decisiones necesarias para la mejora continua de la organización.

Cohesión // Modelos mentales: La organización no tiene definida sus áreas lo que le dificulta la comunicación asertiva entre los actores de cada proceso, de igual manera la alta dirección no tiene una relación directa con los mismos ocasionándoles inconvenientes en la resolución de problemas y la integración de las áreas. 
Ciudadanía // Visión compartida: Los colaboradores que hacen parte de la empresa Componentes S.A.S., están comprometidos en el crecimiento de la organización, pero hace falta que la empresa implemente formas de divulgación a todas las partes interesadas sobre lo que se espera en el futuro para la empresa.

Desempeño // Aprendizaje en equipo: La falta de medición del clima laboral en la empresa no permite conocer el estado actual de la satisfacción de los colaboradores al realizar su trabajo, no se identifican las acciones de mejora que desde la ejecución del proceso se revelan, por lo anterior es posible que no cumplan con los resultados esperados.

\subsection{Interpretación de herramienta de Obstáculos de aprendizaje}

Por medio de la herramienta obstáculos de aprendizaje, la empresa Componentes S.A.S., se sitúa en el $61 \%$ referente a las situaciones que se presentan en la organización, es decir, en la organización existen obstáculos de aprendizaje, pero pueden controlarse.

Basados en la herramienta de aplicación podemos evidenciar que para el área administrativa se encuentran tres obstáculos: audiencia, supersticioso y ambiguo. Estos tres aspectos muestran la falta de entendimiento y conceptualización por parte de los colaboradores en las funciones que desempeñan, lo que puede causar retrocesos; adicionalmente, no se realizan las mediciones que son importantes para validar el cumplimiento de los objetivos propuestos por la organización y lo cual facilita la toma de decisiones, tampoco se tiene en cuenta la retroalimentación que otras áreas puedan aportar, por lo que los cambios o mejoras se basan en lo encontrado por los integrantes de la misma área.

Para el área administrativa financiera se identificaron cinco obstáculos: rol, audiencia, supersticioso, ambiguo y superficial. Estos obstáculos evidencian la falta de asignación en los roles y responsabilidades, lo que dificulta la integración de los procesos, esto conlleva a no tener una medición y control que facilite la toma de decisiones, además tampoco cuenta con una adecuada socialización de los procesos, por lo que las personas crean su propia metodología y si deciden irse de la empresa se llevan su conocimiento, por lo que es necesario empezar de cero cuando llega otro colaborador a realizar la misma labor.

Por otro lado, en el área comercial y de supervisión se identificaron los siete obstáculos: rol, audiencia, supersticioso, ambiguo, superficial, fragmentado y oportunista. Se evidencia que para estas áreas igualmente afecta lo anteriormente mencionado en cuanto a la asignación de 
roles y responsabilidades para tener un orden adecuado en la ejecución de los procesos, adicionalmente, la falta de medición y toma decisiones. Pero como punto adicional tenemos la necesidad de contar con capacitaciones adecuadas para comprender el correcto manejo de los procesos en la organización y tener una curva de aprendizaje continua que facilite la retroalimentación entre los colaboradores y la alta gerencia.

\subsection{Interpretación de la herramienta Modelo de Sistema Viable MSV}

El Modelo de Sistema Viable MSV es una excelente herramienta de diagnóstico que ayuda a diseñar y mejorar el sistema de una organización.

Es importante entender en todo sentido el estado real de una organización, con el fin de no causar lesiones que perjudiquen el sistema actual. Esta herramienta proporciona mucha ayuda al diseño siempre y cuando se entienda cada función. Cuando se aplica esta herramienta de diseño se logra obtener una visión más acertada de la realidad; sin embargo, es importante siempre tener en cuenta el sentido del modelo como un todo y su entorno en general, esto con el fin de obtener los resultados reales y sus objetivos.

En referencia al Modelo de Sistema Viable para la empresa Componentes S.A.S., se evidencia que tiene establecidos algunos de sus procesos misionales con sus respectivas descripciones en cuanto a las funciones a desempeñar por los colaboradores, pero no tiene diseñado un manual de funciones, procesos y procedimientos que brinden una guía al colaborador para desempeñar óptimamente las funciones que se le haya asignado.

Por otro lado, la organización actualmente no desarrolla ninguna auditoría que permita recolectar información e identificar los riesgos y oportunidades que se tienen, para posterior a ello realizar un seguimiento y medición a los cambios que se puedan implementar.

Debido a que no existe seguimiento y medición, la alta dirección no tiene información que permita definir y establecer un horizonte, ni se evidencia un compromiso al cambio, pudiendo ubicar las falencias y poder establecer mejoras que permitan atacarlas.

Gracias a esto, igualmente ninguno de sus procesos cuenta con algún avance de innovación desde la fundación de la organización. 


\section{PLAN DE MEJORA}

\subsection{Plan de mejora articulación ISO HSEQ}

En la actualidad, es fundamental gestionar una organización de manera sistemática y en tiempo real, impulsando la mejora continua en el desempeño de la misma. Un factor clave para que esto sea exitoso es la integración de las diferentes variables tales como Calidad, Gestión Ambiental y Seguridad y salud en el Trabajo, con el fin de eliminar cualquier posibilidad de riesgo, ya que las mismas contribuyen a la mejora de la productividad de la empresa, incrementan la capacidad de respuesta y la recuperación ante cualquier posible situación o incidente; así mismo, disminuyen los riesgos de fallas, reprocesos, accidentes, entre otros. Otro factor es la estandarización de los procedimientos que contribuye a la modernización de la organización. La articulación de un sistema ISO HSEQ no deben ser una opción de incorporación en una organización, sino que debe ser parte esencial de la gestión empresarial con el fin de potenciar la eficiencia y efectividad de los procesos.

El objetivo principal para una adecuada articulación, es analizar cuáles son las claves fundamentales para integrar correctamente un sistema de gestión, entre ellas tenemos: - Qué sistemas se desean integrar.

- Designar personal responsable del proyecto; y al tratarse de una articulación de tres normas, crear una especie de comité de seguimiento, coordinación y control de cada uno de los procesos, áreas o sistemas que se vayan a integrar.

- Establecer un cronograma o plan de trabajo donde se estipulan plazos de ejecución y entrega, la designación de responsables y la documentación necesaria o que se creará a partir de la ejecución del proyecto de articulación.

- Por último y no menos importante es la comunicación de la nueva estructura del

sistema a todo el personal.

Para comenzar a articular el sistema HSEQ es importante conocer los requisitos generales de las normas, tanto internos como externos, para ello existen varias herramientas básicas, eficientes, que analizan los diferentes contextos y que permiten una mayor comprensión, tales como:

- Análisis PESTEL (Político, Económico, Social, Tecnológico, Ecológico, Legal) 
- Modelo de las 5 fuerzas de Porter

- Análisis DOFA

- Modelo de las $5 \mathrm{~S}$

Por otro lado, el conocimiento en absoluto de cada uno de los procesos (estratégicos, misionales y de apoyo) y las actividades de la organización es fundamental ya que permitirá tener una idea más clara en donde va a estar inmersas, tanto las cuestiones de calidad directamente relacionadas con el producto, si la organización se dedica a la transformación de la materia prima, o simplemente a la comercialización o venta de algún producto o servicio; así como las cuestiones relacionadas con la gestión ambiental y aquellas relacionadas directamente con el recurso humano, con el fin de dar un mayor cumplimiento a los debes de las normas.

De la misma forma, es importante identificar los objetivos del Sistema HSEQ, los cuales deben ser concernientes con la política del sistema (Calidad, Medioambiente y SST), esto se puede realizar utilizando el método SMART (Especifico, Medible, Alcanzable, Relevante y Limitado en el Tiempo) (EscuelaEuropeaExcelencia, 2018) que permite obtener una postura más clara para la planificación de todo el Sistema de Gestión Integral HSEQ.

Por otra parte, la organización debe establecer roles, responsabilidades y autoridades, esto para tener un orden adecuado y manejo correcto de la implementación del Sistema de Gestión Integral. También, la organización debe asegurarse de informar y divulgar esta asignación, verificando que los procesos se están llevando a cabo, retroalimentando los constantes cambios, cuáles son las necesidades que se presentan, los posibles riesgos y que cambios se pueden o se deben implementar.

Para la identificación de los procesos es recomendable realizar una estructura organizacional que permita la identificación del nivel jerárquico de la organización, establecer autoridad e identificar las áreas o departamentos de la empresa; esta estructura organizacional conllevará a un análisis más concreto de las actividades primarias, secundarias y hasta terciarias de los procesos y procedimientos inmersos en los diferentes cargos. La empresa se encargaría de definir qué tipo de estructura organizacional es la apropiada según estrategia organizacional, ya sea lineal, funcional, matricial, entre otros, lo que permitirá realizar la caracterización de los procesos. 
Ahora veamos, para la caracterización de los diferentes procesos, analizar de manera concreta los elementos que lo componen de principio a fin, siempre teniendo en cuenta los principales de operación y los que hacen parte esencial para su buen funcionamiento (estratégicos, misionales y de apoyo). Dentro de esta actividad, se identifica las diferentes fases de cada proceso, desde las entradas requeridas, sus actividades y la salida esperada; además, de los controles e indicadores que se asocian al buen desempeño del proceso.

\begin{tabular}{|c|c|c|c|c|}
\hline \multicolumn{3}{|c|}{ CARACTERIZACIÓN DE PROCESOS } & \multirow{2}{*}{\begin{tabular}{|l|} 
Código \\
Versión \\
\end{tabular}} & \multirow{2}{*}{\begin{tabular}{|l} 
XX-001 \\
1
\end{tabular}} \\
\hline & & & & \\
\hline & & & Fecha & D-M-A \\
\hline \multicolumn{5}{|l|}{ PROCESO } \\
\hline \multicolumn{5}{|l|}{ OBJETIVO } \\
\hline \multirow[t]{4}{*}{ ENTRADAS } & ACTIVIDADES & SALIDAS & \multirow{4}{*}{\multicolumn{2}{|c|}{$\begin{array}{l}\text { REQUISITOS NORMAS } \\
9001: 2015 \text { Y 14001:2015: }\end{array}$}} \\
\hline & 1. & & & \\
\hline & 2. & & & \\
\hline & 3. & & & \\
\hline \multicolumn{3}{|c|}{ INFORMACIÓN DOCUMENTADA: } & \multicolumn{2}{|l|}{ KPI's } \\
\hline
\end{tabular}

Figura 3 Ejemplo de caracterización de procesos

En términos de que todo el sistema debe ser trazable y medible, es necesario garantizar indicadores de desempeño con el que se pueda realizar análisis y evaluación constante al mismo. Indicadores de rendimiento en producción, satisfacción del cliente, ventas, de proveedores, entre otros.

La actividad principal de la empresa Componentes S.A.S., es la comercialización de línea de autopartes, ferreterías y elementos de protección personal EPP'S, por lo que debe garantizar el buen manejo de los inventarios, stocks, entregas, suministros disponibles, despachos, entre otros. Just in Time es una metodología que sirve para organizar la producción de las organizaciones, desde la compra del producto hasta su distribución (por sus siglas en español Justo a Tiempo), esta metodología permite que los materiales desde la producción hasta el producto terminado lleguen a su destino "cuando sea necesario, ni antes ni después"

(Isotools.org, 2020).

El mayor reto al que se exponen las pequeñas y medianas empresas es la adaptación a nuevas tecnologías existentes en el mercado, ya que, aunque son verdaderamente eficientes, su elevado costo dificulta la implementación. Herramientas como los sistemas ERP sistemas de planificación de recursos empresariales (por sus siglas en inglés enterprise resource 
planning) se convierten en un gran aliado para el apropiado manejo, control y seguimiento y automatización de todos procesos de una compañía, desde el aprovisionamiento hasta la disposición final del producto o servicio; adicionalmente, la documentación, indispensable para el cumplimiento del requisito 7.5 Información Documentada de las tres normas, se encontrará en tiempo real y segura por su almacenamiento en la nube.

Estos sistemas se pueden adaptar fácilmente a la actividad realizada por la empresa, ya que la estructura de software permite la configuración o parametrización de los diferentes módulos que solicita la organización; la estructura básica de un ERP se caracteriza por contar con los 5 módulos más convencionales dentro de una organización, tales como Producción, Ventas y Finanzas; sin embargo, la empresa puede solicitar otros de acuerdo a su necesidad.

- Actividades relacionadas al plan

1. Análisis del contexto estratégico (determinación de factores externos e internos).

2. Sensibilización y capacitación sobre la implementación HSEQ a todo el personal.

3. Revisión de objetivos concernientes al SGI, gestión y planificación para lograrlos.

4. Revisión de recursos y competencias.

5. Levantamiento de información documentada.

6. Actualización de indicadores (revisión de evaluación de desempeño).

7. Generación programa de auditoría.

\subsection{Plan de mejora arquetipos}

En la empresa Componentes S.A.S., los arquetipos organizacionales deben identificarse con el fin de evaluar la oportunidad de mejora.

"La cultura organizacional crea en el grupo un orden social, genera continuidad y desarrolla una identidad colectiva y sentimiento de pertenencia. Las personas que pertenecen a una cultura organizacional comparten, a través de la experiencia colectiva y repetidas interacciones sociales a lo largo del tiempo"1

${ }^{1}$ León, R. A. (2001). Cultura organizacional y liderazgo: Reflexiones sobre algunos resultados de entidades bancarias. Revista de Psicología del Trabajo y de las Organizaciones, Pág. 157. 
El modelo que se propone para que la empresa Componentes SAS se enfocara en la administración estratégica2

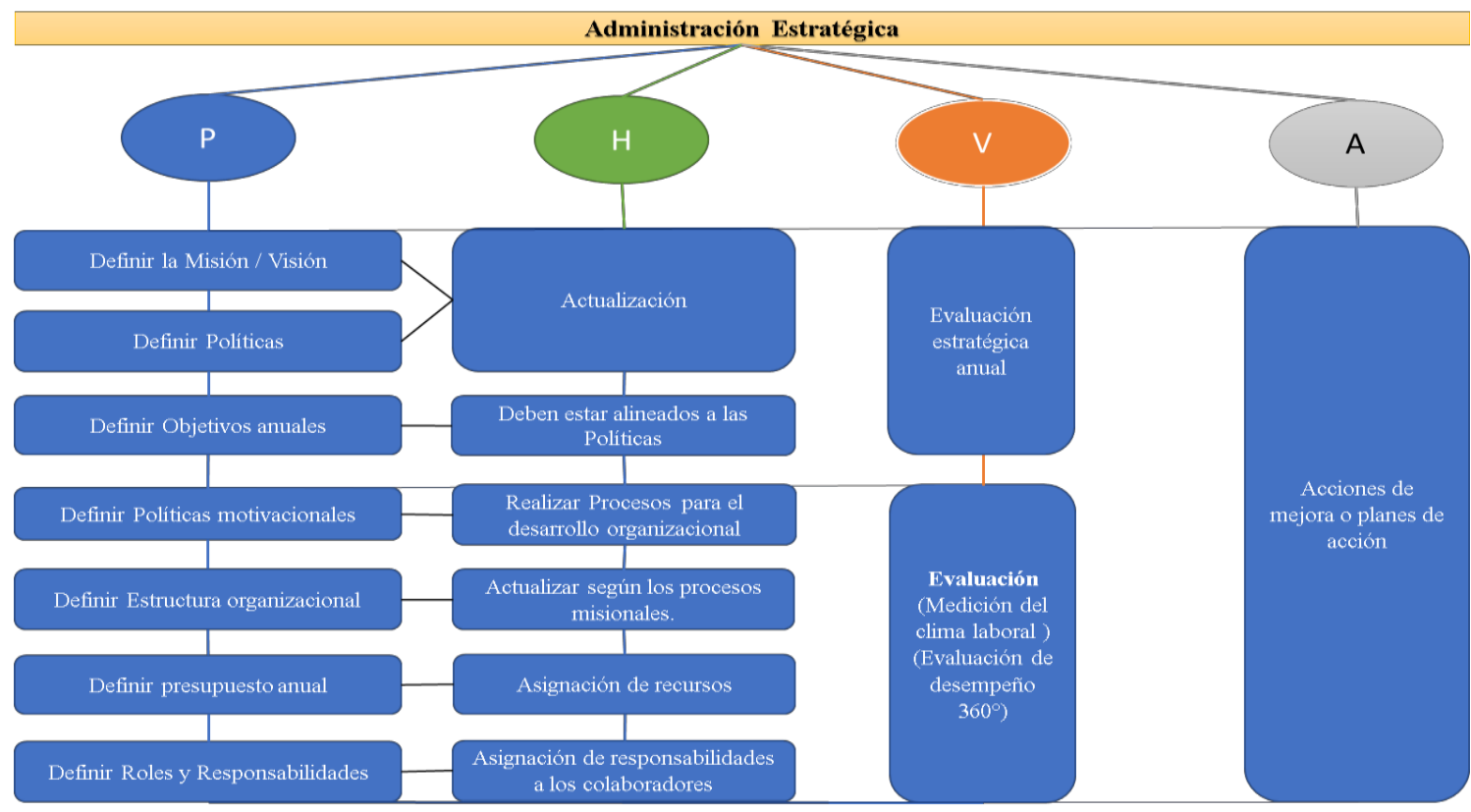

Figura 4 Administración Estratégica (fuente propia)

- Actividades relacionadas al plan

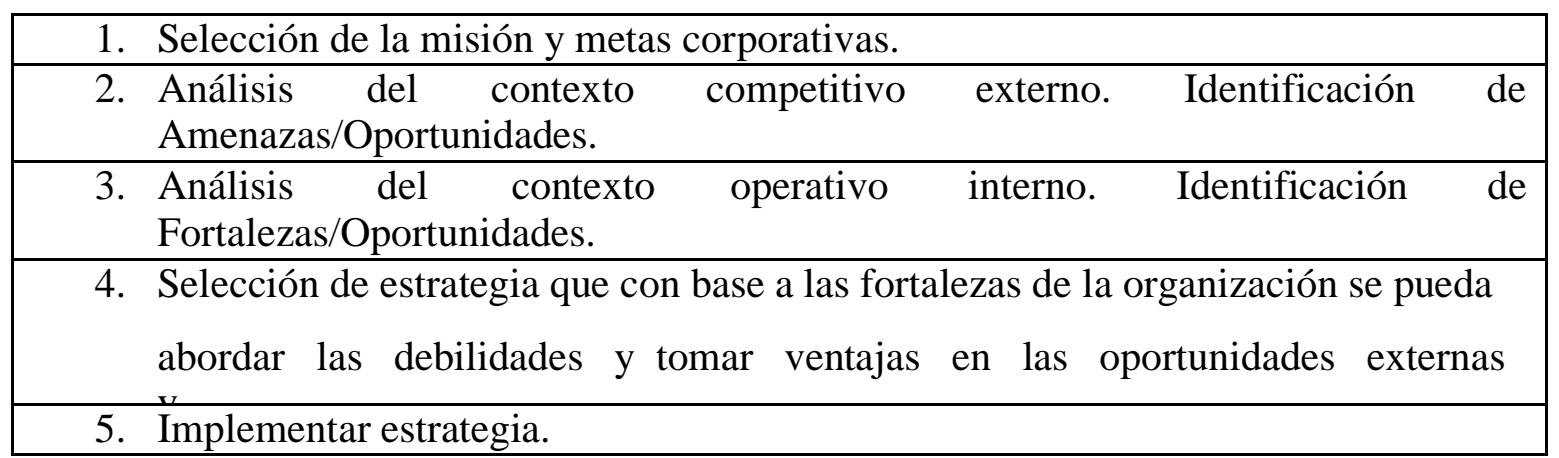

\subsection{Plan de mejora obstáculos de aprendizaje}

Para garantizar el éxito de los diferentes procesos de la organización, es fundamental adquirir compromiso con aquellas cuestiones relacionada con los obstáculos de aprendizaje, ya que esto, en muchas ocasiones, dificulta los procesos. 
Es importante la capacitación constante del personal de organización, para que sus competencias y habilidades se desarrollen y garanticen que los procesos de la organización tienen una responsabilidad por parte del empleado; la capacidad de poder admitir que existe una falencia o poco conocimiento sobre algo, es fundamental para implementar acciones preventivas y correctivas frente los procesos y al personal.

La resistencia al cambio, debe ser tratada con compromiso por la alta dirección y por parte de los líderes de la organización. Una manera efectiva de hacerlo, es implementando el Modelo Feedback (retroalimentación) que identifica las cuestiones que no son efectivas de comunicación dentro de la organización, permitiendo garantizar la continuidad del proceso comunicativo de manera asertiva, logrando cambios dentro de la organización, avanzando en el compromiso y los acuerdos de cada uno de los colaboradores para la ejecución correcta de las tareas, dando y recibiendo el resultado adecuado.

La aplicación del Modelo de aprendizaje individual OADI (Observar - Evaluar - Diseñar Implementar por siglas en español) que facilita el aprendizaje organizacional e influye directamente en las acciones que se desarrollan a diario por parte del individuo, cambiando su creencia de la forma correcta de desarrollar dicha actividad generando un ciclo de aprendizaje, una evaluación por parte de todas las áreas y así crear una correcta forma de hacer las cosas.

- Actividades relacionadas con el plan

1. Capacitar en la toma de conciencia para el compromiso estratégico con el

2. Implementar talleres de liderazgo y aprendizaje individual

3. Capacitar a las áreas estratégicas de la organización en temas relacionados con la

4. Implementar estrategias de retroalimentación para el personal.

\subsection{Plan de mejora Modelo de Sistema Viable MSV}

Implementar un sistema a través de esta herramienta, como instrumento de diseño, es vital para proponer cambios factibles dentro de la organización. Es importante determinar, a través 
de esta metodología, la construcción del modelo teniendo en cuenta sus fases; implementación, Coordinación, Control, Inteligencia y Política.

Se identifica como primera fase de Implementación el análisis del estado real de la organización; con qué cuenta, qué tiene implementado, ha identificado sus actividades esenciales, etc. En la Fase de Coordinación se analiza la interacción de cada uno de los procesos y su coordinación, verificando la eficiencia de la administración de los recursos, si existe la mejora continua del proceso y la efectividad en la asignación de las tareas. Se evidencia que la organización Componentes S.A.S. responsabiliza a una sola persona en varias tareas, lo que no permite garantizar efectividad en las mismas y al mismo tiempo retraso y desorden. En la Fase de Control se debe planear o establecerse todo aquello que afecte directamente el proceso a nivel general de la organización, tales como inventarios, manejo de la información, atención al usuario/cliente y el uso de los recursos y tecnología.

- Actividades relacionadas con el plan

1. Análisis de estado real de la organización (Fase 1).

2. Revisión de la interacción de cada uno de los procesos y su coordinación. (Fase 2)

3. Revisión del recurso físico y de personal disponible.

4. Analizar todo aquello que afecte los procesos de la organización (Fase de Control).

\section{CONCLUSIONES}

Actualmente no existe un requisito universal que explique cuál es la forma ideal de integrar las Normas relacionada con Sistemas de Gestión Integral, tales como NTC ISO 9001:2015,

14001:2015 y 45001:2018; sin embargo, la integración de estos estándares se puede lograr a través de guías prácticas o en función a requisitos comunes entre las Normas. La estructura de alto nivel de las Normas NTC ISO hace posible la uniformidad en las mismas, lo que pretende que exista una coherencia entre ellas, permitiendo que las organizaciones puedan integrarlas a su Sistema de Gestión de Calidad de una forma más sencilla, siempre con la independencia del ámbito de cada una, es decir sin llegar a perder su finalidad para cada entorno.

Las Normas NTC ISO 9001:2015 y NTC ISO 14001:2015, aunque no son un requisito obligatorio, son de las Normas más populares en términos de Calidad y gestión Ambiental, pues según la Revista Dinero, Colombia es el tercer país en Latinoamérica con mayor número 
de certificados bajo estas Normas por encima de Chile y México, demostrando que existe un alto índice de competitividad, Calidad y gestión Ambiental dentro del mercado interno. Por otro lado, el Decreto 1072 de 2015 se convierte en un requisito obligatorio para que las organizaciones, independientemente de su tamaño cuenten con su sistema de seguridad y salud en el trabajo SG-SST, donde en algunos aspectos se alinean con la Norma NTC ISO 45001: 2018.

La implementación de un Sistema de Gestión Integral ha tomado importancia en las organizaciones, ya que al encontrarse en un mercado global cada vez más competitivo hace necesaria la búsqueda de mejores herramientas estratégicas para logarlo. Otro factor fundamental y uno de los de mayor interés, es la optimización de recursos y en ejecución de esto ser más rentable. Por otro lado, los requisitos legales (mayormente en Medio Ambiente y SST) hacen que las organizaciones cumplan obligatoriamente con estas exigencias para operar sin importar el objeto social o actividad.

El contexto actual y las organizaciones han notado que ya no es suficiente producir un producto de calidad, sino que dentro de su cadena de procedimientos reconocen el rol tan importante que cumplen, por un lado, dentro del medio ambiente, la gestión adecuada de sus impactos y aspectos ambientales, y por el otro, gestionando de la mejor manera los riesgos a nivel de seguridad y salud en el trabajo, ya que con esto demuestran el nivel de competencia con el que cuentan y el compromiso con todas la partes interesadas.

La identificación de requisitos comunes y la interrelación entre las Normas, se convierte en una necesidad para alinear y garantizar el cumplimiento total o parcial de las mismas siempre y cuando se conviertan en una estratégica que fortalezca las capacidades y logre los objetivos dentro de la organización desde cualquier perspectiva: mercado, competencia, cliente interno y externo y hasta resultados financieros favorables; ya que, el sentido de esta tres Normas es garantizar la eficacia (cumplimiento de objetivos) y eficiencia (conseguir los resultados planificados) siempre en pro de la mejor continua.

El compromiso para el Sistema de Gestión Integral es fundamental, no solo para aquellos que tienen a cargo su creación y mantenimiento, sino también de todos los relacionados en cualquier proceso, ya sean Misional de Apoyo o Estratégicos, puesto que de eso depende que el sistema se mantenga a través de los años y además que se garantice el buen funcionamiento del mismo. 
Otro factor fundamental es el pensamiento sistémico con respecto a la interpretación adecuada de cualquier entorno, esto es vital para desarrollar metodologías que permitan dar solución de cualquier problemática desde diferentes perspectivas. Entender la interacción que existe entre los diferentes sistemas, los eventos que se crean a partir de esto y las características que los definen, y a partir de ellos comprender la realidad con el fin de establecer soluciones adecuadas y duraderas a través del tiempo.

La dinámica sistémica permite una mejor comprensión de la realidad en los diferentes factores presentes en el entorno empresarial, tales como culturales, tecnológicos, ambientales y sociales; cuando una organización además de controlar, responde a los cambios producidos dentro de su propio sistema, pero además responde a entornos macro o sistemas externos que se interrelacionan entre sí, logra y crea una ventaja competitiva y diferenciadora en el mercado global.

A su vez, la aplicación de herramientas que fortalezcan la mejora continua en los diferentes procesos inmersos dentro de un sistema, contribuye a responder a cualquier suceso, pero además a tener control dentro del mismo. En la actualidad, existen múltiples herramientas y técnicas que se enfocan en la mejora continua de aquellas áreas críticas, capaces de generar altos índices de riesgo y son vulnerables al deterioro de una organización; la capacidad de identificar cada una de ellas, a través del Sistema de Gestión Integral contribuye a las organizaciones a intervenir cuando esto sea necesario. Según la Norma Técnica Colombiana NTC ISO 9000:2015 la Mejora continua "es una actividad recurrente para mejorar el desempeño" que, dicho en otras palabras, se convierte en sinónimo de supervisar, controlar, documentar e intervenir, todo esto con un enfoque basado en el ciclo PHVA (Planificar, Hacer, Verificar y Actuar), complemento fundamental para el pensamiento sistémico.

En conclusión y a grosso modo, las diferentes normas expuestas en éste capítulo, herramientas y técnicas aplicables a un SGI se fundamentan bajo una estructura o filosofía con el fin de poder interrelacionar una con la otra $y$, en ese sentido, se vuelve vital el compromiso de los actores, que, no importando su nivel jerárquico dentro de la organización, deben tener no solo con su implementación sino con su sostenimiento a través de los años, lo cual podría convertirse en el mayor reto organizacional.

Integrar de forma activa todos los colaboradores es una estrategia para las organizaciones, donde se cree una cultura organizacional capaz de identificar, moldear y comunicar cualquier situación positiva o negativa que permitan el crecimiento constante de las organizaciones. En otras palabras, generar ambientes laborales positivos que contribuyan al crecimiento 
organizacional y profesional, ya que por muy buenas que sean estas herramientas, su utilidad radica en la habilidad y compromiso de quien la utiliza.

La evolución de una organización debe ir enfocada en búsqueda de la excelencia, reconociendo los conceptos y requisitos mínimos de las normas a implementar para luego ir incorporando directrices guías para la mejora del desempeño de la organización como la NTC ISO 9004:2015 y para hacer esto efectivo el alto compromiso, la alineación de esfuerzos de todos los colaboradores y de las áreas de la organización deben trascender en el tiempo, no puede ser algo temporal; ser capaces de tomar decisiones, actuar y trabajar en pro de lograr la visión, objetivos y metas para la mejora continua de la organización.

El objetivo de un sistema HSEQ es permitir la interacción de los sistemas, que a su vez están interrelacionado y que tiene como fin establecer políticas y objetivos para cada entorno Calidad, Medio Ambiente y Seguridad y Salud en el Trabajo. La normalización es fundamental para establecer un orden en cada actividad con el fin de evaluar las no conformidades que puedan existen dentro del sistemas de gestión que permitirá ofrecer un mejor servicio en todo contexto, desde tener una excelente identidad corporativa hasta identificar las actividades que no son necesarias en la organización. 
ANEXOS

1. Lista de Verificación NTC ISO 9001:2015

2. Lista de Verificación NTC ISO 14001:2015

3. Lista de Verificación NTC ISO 45001:2018

4. Lista de Verificación Modelo de Sistema Viable

5. Herramienta para identificar Arquetipos Organizaciones

6. Obstáculos de Aprendizaje

7. Carpeta (Planes de Trabajo)

REFERENCIAS BIBLIOGRÁFICAS

1. Contreras Vallecilla, J, A., Moreno Huertas, W, A., \& Soriano Novoa, D, M. (21 de 11 de 2020). IMPLEMENTACIÓN DE LOS SISTEMAS DE GESTIÓN DE CALIDAD SEGÚN LA NTC ISO 9001:2015. Semillero de investigación HSEQ 2020. Bogotá, Cundinamarca, Colombia: FUAC.

2. Camberos Ortiz, M, E. \& Higuera Barbosa, G, S. (21 de 11 de 2020). PROPUESTA DE ARTICULACIÓN ENTRE LA NORMA ISO 14001: 2015 Y LA LEGISLACIÓN COLOMBIANA. Semillero de investigación HSEQ 202. Bogotá, Cundinamarca, Colombia: FUAC.

3. Bareño Moreno, H, K., López Rodríguez, Y, M., \& Prieto Ramírez, L, F. (21 de 11 de 2020). ARTICULACIÓN DE LA NORMA TÉCNICA COLOMBIANA (NTC) NTC ISO 45001 Y LA LEGISLACIÓN COLOMBIANA (DECRETO 1072, CAPÍTULO 6). Semillero de investigación HSEQ 2020. Bogotá, Cundinamarca, Colombia: FUAC.

4. Guevara Cerón, G, R., Jiménez Mora, M, L., \& Rodríguez Moreno, J, T. (21 de 11 de 2020). UNA HERRAMIENTA PARA EL DIAGNÓSTICO DE OBSTÁCULOS QUE INHIBEN EL APRENDIZAJE EN LAS ORGANIZACIONES. Semillero de investigación HSEQ 2020. Bogotá, Cundinamarca, Colombia: FUAC. 
5. Maldonado Méndez, J, S., Suárez Russi, Y, M., \& Vargas Medina, L, N. (21 de 11 de 2020). ARQUETIPOS ORGANIZACIONALES A PARTIR DE RAÚL ESPEJO Y PETER SENGE. Semillero de investigación HSEQ 2020. Bogotá, Cundinamarca, Colombia: FUAC.

6. "Modelo de Sistema Viable aplicado a una empresa de transporte de carga: ENTREKARGA" (Margarita María Bastidas y Laura Carolina Murillo, 2013): https://repositorio.uniandes.edu.co

7. "El Modelo de Sistema Viable: Un instrumento para la Organización Efectiva" (Norlando Sanchez Rueda): https://journal.universidadean.edu.c 